\title{
Pretreatment with statins enhances myocardial protection during coronary revascularization
}

Harold L. Lazar, MD, Yusheng Bao, MD, Yu Zhang, BS, and Sheilah A. Bernard, MD

Objective: This experimental study was undertaken to determine whether pretreatment with statins would enhance myocardial protection and minimize ischemic injury during revascularization of acutely ischemic myocardium.

Methods: In 20 pigs the second and third diagonal arteries were occluded for 90 minutes, followed by 45 minutes of blood cardioplegic arrest and 180 minutes of reperfusion. Ten pigs received atorvastatin ( $40 \mathrm{mg}$ orally every day) for 21 days before surgical intervention; 10 others received no statins. Ischemic damage was assessed on the basis of the need for cardioversions for ventricular arrhythmias, regional wall-motion scores $(4=$ normal to $-1=$ dyskinesia $)$ were determined by means of 2-dimensional echocardiography, endothelial function was assessed on the basis of bradykinin-induced coronary artery relaxation, and infarct size was calculated by determining the area of necrosis to the area of risk by means of histochemical staining. Results are given as means \pm SE.

Results: Statin-treated animals required fewer cardioversions $(0.11 \pm 0.01$ vs $2.87 \pm 0.20, P=.0001)$, had improved wall-motion scores ( $2.81 \pm 0.10$ vs $1.52 \pm$ $0.08, P=.01)$, had lower infarct size $(21 \% \pm 2 \%$ vs $41 \% \pm 2 \%, P=.0001)$, and had more complete coronary artery relaxation $(34 \% \pm 5 \%$ vs $8 \% \pm 4 \%, P=.01)$. Total serum cholesterol levels were similar between the groups $(62 \pm 5 \mathrm{mg} / \mathrm{dL}$ for statin-treated animals vs $68 \pm 5 \mathrm{mg} / \mathrm{dL}$ for non-statin-treated animals, $P=.30$ ).

Conclusions: Pretreatment with statins enhances myocardial protection during revascularization by means of mechanisms that are independent of their cholesterollowering properties.

From the Department of Cardiothoracic Surgery, The Boston Medical Center and The Boston University School of Medicine, Boston, Mass.

Supported in part by a research grant from Pfizer, Inc.

Read at the Eighty-second Annual Meeting of The American Association for Thoracic Surgery, Washington, DC, May 5-8, 2002.

Received for publication May 29, 2002; revisions requested Aug 16, 2002; revisions received Aug 22, 2002; accepted for publication Aug 26, 2002.

Address for reprints: Harold L. Lazar, MD, Department of Cardiothoracic Surgery, Boston Medical Center, 88 E Newton St, Suite B404, Boston, MA 02118 (E-mail: harold.lazar@bmc.org).

J Thorac Cardiovasc Surg 2003;125: 1037-42

Copyright $(92003$ by The American Association for Thoracic Surgery

$0022-5223 / 2003 \$ 30.00+0$

doi: $10.1067 / \mathrm{mtc} .2003 .177$

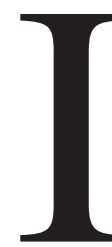

nhibitors of 3-hydroxy-3-methylglutaryl coenzyme A reductase, collectively known as statins, have been shown to minimize myocardial ischemic events in patients with hypercholesterolemia without manifestations of cardiovascular disease ${ }^{1,2}$ and in those patients who have already undergone revascularization procedures, such as coronary artery bypass grafting $(\mathrm{CABG})$ and percutaneous transluminal coronary angioplasty (PTCA). ${ }^{3-5}$ Hypercholesterolemia results in increased platelet reactivity, endothelial dysfunction, atherosclerotic plaque formation, and, ultimately, plaque disruption, which results in acute coronary syndromes. Statins, by lowering serum cholesterol levels, reduce blood thrombogenicity and improve endothelial function, thereby improving clinical outcomes. However, recent experimental and clinical studies suggest that the beneficial effects of statins might extend beyond their ability to reduce serum cholesterol levels. ${ }^{6-10}$ In the Myocardial Ischemia Reduction with Aggressive Cholesterol Lowering (MIRACL) trial, high doses of atorvastatin administered to patients with unstable angina and non-Q-wave myocardial infarction (MI) significantly reduced the incidence of recurrent anginal episodes within several weeks. ${ }^{11}$ Furthermore, at baseline, the low-density lipoprotein (LDL) levels in both groups were normal (123-125 mg/dL), suggesting that the beneficial effects of statin therapy might occur in the presence of normal serum cholesterol levels. Recent 
experimental studies in animals subjected to acute ischemic injury also showed decreased damage with statins in the presence of normal serum cholesterol. ${ }^{7-10}$

This experimental study was therefore undertaken to investigate whether statin therapy might also have a role during coronary revascularization. Specifically, we sought to determine whether pretreatment with atorvastatin would enhance myocardial protection in pigs undergoing surgical revascularization for acutely ischemic myocardium and whether the improved outcomes could be correlated with reductions in serum cholesterol levels.

\section{Methods \\ Randomization}

Twenty adult pigs $(35-40 \mathrm{~kg})$ were fed a standard chow diet for 3 weeks. The animals were randomized to statin-treated or nonstatin-treated groups by means of a sealed envelope that assigned them to receive a 21-day supply of either atorvastatin $(40 \mathrm{mg}$ orally every day) or a placebo. All animals completed the 3-week pretreatment phase and were entered into the study.

\section{Preparation}

The pigs were premedicated with intramuscular injections of ketamine $(15 \mathrm{mg} / \mathrm{kg})$ and xylazine $(0.5 \mathrm{mg} / \mathrm{kg})$, anesthetized with $\alpha$-chloralose (75 mg/kg), intubated, and placed on positive-pressure ventilation. A mediasternotomy was performed, and the animals received systemic heparinization $(3 \mathrm{mg} / \mathrm{kg}$ ). The second and third diagonal coronary arteries just distal to the takeoff of the left anterior descending artery were occluded with snares for $90 \mathrm{~min}-$ utes. The pigs were then placed on cardiopulmonary bypass, and the hearts were arrested with multidose, antegrade-retrograde, cold blood cardioplegia (potassium $25 \mathrm{mEq} / \mathrm{L}$; hematocrit 20\%; pH 7.6; temperature $4^{\circ} \mathrm{C}$ ) supplemented with topical hypothermia for 45 minutes. The aortic crossclamp was then removed, the coronary snares were released, and all hearts were perfused in normal sinus rhythm for 180 minutes on cardiopulmonary bypass with a mean aortic pressure of $60 \mathrm{~mm} \mathrm{Hg}$ at $37^{\circ} \mathrm{C}$.

\section{Experimental Groups}

During the 3 weeks before the operation, the animals were divided into 2 groups. In the non-statin-treated group 10 animals received only a placebo with their chow. In the statin-treated group 10 animals received atorvastatin, $40 \mathrm{mg}$ orally every day, with their chow. This dose of atorvastatin has been shown to reduce ischemic events in clinical studies after an acute MI. ${ }^{11}$

\section{Measurements and Statistical Analyses}

Electrocardiographic leads were placed to measure heart rate. Left ventricular end-diastolic pressure was recorded with a piezoelectric Mikro-Tip catheter pressure transducer (Millar Instruments Inc, Houston, Tex) inserted through the left ventricular apex.

Electrical cardioversion was used to treat ventricular fibrillation and ventricular tachycardia, which was either unresponsive to intravenous lidocaine or caused a clinically significant $(>20 \mathrm{~mm}$ $\mathrm{Hg}$ ) decrease in systolic blood pressure. Serum concentrations of total cholesterol, LDL cholesterol, high-density lipoprotein cholesterol, and triglycerides were determined by means of standard enzymatic methods at the Boston Medical Center Central Laboratory.

Echocardiographic short- and long-axis sections obtained from transthoracic echocardiograms were used to define wall-motion changes in the area of risk, as previously described. ${ }^{12} \mathrm{~A}$ wallmotion score (WMS) was derived $(4=$ normal, $3=$ mild hypokinesis, $2=$ moderate hypokinesis, $1=$ severe hypokinesis, $0=$ akinesis, and $-1=$ dyskinesia) to indicate changes in wall motion. The sections were interpreted by an experienced echocardiographer (S.A.B.) in a blinded fashion, and the scores were averaged for the coronary occlusion and reperfusion periods for all experimental groups.

Infarct size was assessed by determining the areas of necrosis to areas of risk by using histochemical staining techniques with triphenyltetrazolium chloride, as previously described. ${ }^{12}$ Stained myocardial slices were planimetered to obtain the area of risk compared with the total left ventricular mass and the percentage area of infarction in that area of risk.

Epicardial vascular relaxation was assessed by using standard organ chamber methodology. A segment of the second or third diagonal vessel in the area at risk was dissected, cut into rings, and suspended in organ chambers with oxygenated Krebs buffer at $37^{\circ} \mathrm{C}$. Ring tension was determined by using a force-displacement transducer (Grass Instruments, Inc, West Warwick, RI) attached to each tensiometer apparatus and recorded on MacLab recording software. The rings were allowed to equilibrate at a passive tension of 2 to $3 \mathrm{~g}$ for 60 minutes and then contracted with $1 \mu \mathrm{mol} / \mathrm{L}$ prostaglandin $F_{2 x}$ and allowed to stabilize. Once a stable contraction was obtained, coronary vasomotor function was assessed by generating dose-response curves to cumulative concentrations of nitroglycerin $\left(10^{-9}\right.$ to $\left.10^{-5} \mathrm{~mol} / \mathrm{L}\right)$, an endothelial-independent coronary vasodilator, and the calcium ionophore A23187 and bradykinin, endothelial-dependent coronary vasodilators. Relaxation in response to each concentration of the agonist was calculated as the percentage reduction in isometric tension from the tension produced by $1 \mu \mathrm{mol} / \mathrm{L}$ prostaglandin $\mathrm{F}_{2 \mathrm{x}}$. Values were calculated for each experiment, and mean values were computed for the various treatment groups.

All pigs received humane care in compliance with the "Principles of Laboratory Animal Care" formulated by the National Society for Medical Research and the "Guide for the Care and Use of Laboratory Animals" prepared by the Institute of Laboratory Animal Resources and published by the National Institutes of Health (National Institutes of Health publication no. 86-23, revised 1985).

All values are presented as means \pm SE. Nonpaired Student $t$ tests and Wilcoxon Mann-Whitney tests were used to compare measured data between the groups. Differences in hemodynamic measurements between the groups and across time was assessed by means of repeated measurement analysis of variance. Statview 4.5 (Abacus Concepts, Inc, Berkeley, Calif) was used to compute these analyses.

\section{Results \\ Lipid Profiles}

The lipid profiles between the statin-treated and non-statintreated groups are summarized in Table 1. After 3 weeks of statin therapy, there was no difference in total cholesterol, 
LDL, high-density lipoprotein, and triglyceride levels between the groups.

\section{Hemodynamics and Ventricular Irritability During Coronary Occlusion Before Cardiopulmonary Bypass} Table 2 summarizes the hemodynamics and ventricular irritability during the 90 minutes of coronary occlusion before the initiation of cardiopulmonary bypass. Heart rate remained stable in both groups during the period of coronary occlusion. However, mean arterial pressures were lower and left ventricular end-diastolic pressures were higher in both groups compared with preocclusion values. Mean aortic pressure was statistically lower in the statintreated animals after 90 minutes of coronary occlusion (55 \pm 2 vs $62 \pm 7 \mathrm{~mm} \mathrm{Hg}, P=.03$ ). The need for electrical cardioversions for ventricular arrhythmias was much higher in the non-statin-treated animals $(2.87 \pm 0.20$ vs $0.11 \pm$ $0.01, P=.0001)$.

\section{Wall-Motion Scores}

WMSs are summarized in Figure 1. Both groups had decreased wall motion after 90 minutes of coronary occlusion. However, on reperfusion, WMSs were higher in the statintreated animals $(2.65 \pm 0.08$ vs $1.55 \pm 0.10$ at 60 minutes, $P=.01 ; 2.81 \pm 0.10$ vs $1.52 \pm 0.08$ at 180 minutes, $P=$ $.01)$.

\section{Coronary Artery Relaxation}

Figure 2 summarizes endothelial function, as assessed by means of coronary artery relaxation. Endothelial-independent coronary vasodilation in response to nitroglycerin remained intact in both groups $(82 \% \pm 9 \%$ for the non-statintreated group vs $95 \% \pm 7 \%$ for the statin-treated group). Endothelial-dependent vasodilation to both A23187 and bradykinin was markedly decreased in non-statin-treated animals. Statin-treated animals showed better recovery of endothelial-dependent relaxation to both A23187 (20\% \pm $10 \%$ for non-statin-treated animals vs $47 \% \pm 6 \%$ for statintreated animals, $P=.02)$ and bradykinin $(8 \% \pm 4 \%$ for non-statin-treated animals vs $34 \% \pm 5 \%$ for statin-treated animals, $P=.01$ ).

\section{Infarct Size}

The area at risk for necrosis was similar in both groups $(15.5 \% \pm 3.5 \%$ for statin-treated animals vs $18.5 \% \pm 2.3 \%$ for non-statin-treated animals). However, the area of necrosis within the area of risk was lower in statin-treated animals $(21 \% \pm 2 \%$ vs $41 \% \pm 2 \%, P=.0001)$.

\section{Discussion}

Our study has showed that pretreatment with atorvastatin enhances myocardial protection during surgical revascularization of acutely ischemic myocardium. These beneficial effects occurred in the absence of clinically significant
TABLE 1. Lipid profiles

\begin{tabular}{lccc}
\hline Variable & $\begin{array}{c}\text { No statin } \\
(\mathbf{n}=\mathbf{1 0})\end{array}$ & $\begin{array}{c}\text { Statin } \\
(\mathbf{n}=\mathbf{1 0})\end{array}$ & $\boldsymbol{P}$ value \\
\hline Total cholesterol (mg/dL) & $68 \pm 5$ & $62 \pm 5$ & .30 \\
$\quad$ LDL & $38 \pm 5$ & $31 \pm 3$ & .14 \\
HDL & $31 \pm 3$ & $27 \pm 3$ & .50 \\
Triglycerides & $42 \pm 5$ & $32 \pm 10$ & .32 \\
\hline
\end{tabular}

All values are presented as means $\pm \mathrm{SE}$. $H D L$, High-density lipoprotein.

changes in serum cholesterol levels from nontreated animals, suggesting that alternative mechanisms might be responsible for this enhanced myocardial protection. There are several mechanisms by which statins might decrease ischemia-reperfusion injury. These include improved endothelial function, a decreased propensity for platelet thrombus formation, and reductions in the inflammatory process. Nitric oxide (NO) production and its degradation regulates the processes that are responsible for the preservation of endothelial function. Statins are intimately involved with several pathways that can affect NO availability. Statins decrease endothelial cell superoxide production, which inactivates NO. This occurs not only by reducing LDL but by directly preventing the isoprenylation of $\mathrm{P} 21 \mathrm{rac}$, a protein involved in the formation of the superoxide precursor nicotinamide adenine dinucleotide phosphate oxidase. ${ }^{13}$ Statins also upregulate the expression of endothelial NO synthase. ${ }^{10}$ NO synthase activity is inhibited by the protein caveolin 1, which is inhibited by atorvastatin, thus increasing NO synthase activity. ${ }^{14}$ It had been thought that it would take months for these favorable effects on endothelial function to occur. However, statin therapy has been shown to improve flow-mediated brachial artery vasodilatation in clinical studies after only 4 weeks. ${ }^{15}$ By preserving endothelial function, statins might decrease platelet adhesion and the stimulation of plasminogen activator inhibitor, which results in increased thrombogenicity and contributes to ischemic damage during acute coronary syndromes.

Recent experimental studies support the hypothesis that statins exert their anti-ischemic effects in the absence of changes in serum cholesterol and independently preserve endothelial function. Lefer and colleagues ${ }^{9}$ studied the effects of simvastatin administered 18 hours before 20 minutes of ischemia in isolated normocholesterolemic rat hearts. Simvastatin improved left ventricular function, increased coronary blood flow, and endothelial NO production, and significantly reduced leukocyte accumulation in the ischemic myocardium. P-selectin expression and CD18 upregulation were attenuated, and the number of leukocytes adherent to the vascular endothelium was markedly decreased. Hence another mechanism for the cardioprotective effects of statins might be related to their ability to inhibit leukocytes from adhering to the endothelium. Similar find- 


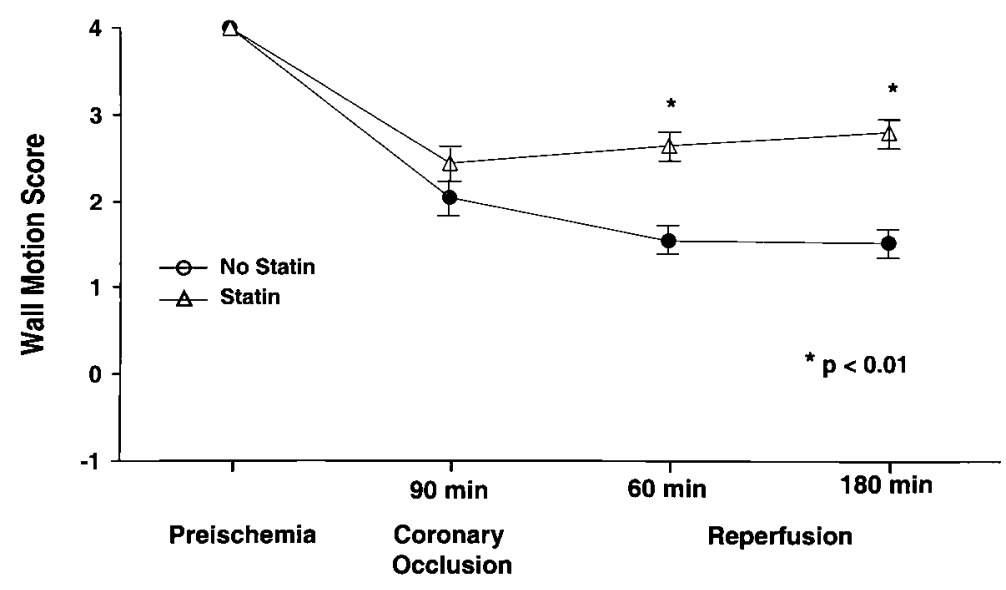

Figure 1. WMSs are higher in the statin-treated animals after reperfusion.

TABLE 2. Heart rate, mean aortic pressure, left ventricular end-diastolic pressure, and ventricular irritability after 90 minutes of coronary occlusion

\begin{tabular}{lcc}
\hline Variable & $\begin{array}{c}\text { No Statin } \\
(\mathbf{n}=\mathbf{1 0})\end{array}$ & $\begin{array}{c}\text { Statin } \\
\text { (n= 10) }\end{array}$ \\
\hline Heart rate (beats/min) & & $115 \pm 7$ \\
$\quad$ Preocclusion & $112 \pm 7$ & $110 \pm 6$ \\
90 min of occlusion & & $74 \pm 6$ \\
Mean arterial pressure (mm Hg) & $62 \pm 7^{*}$ & $55 \pm 2^{*} \dagger$ \\
$\quad$ Preocclusion & & \\
90 min of occlusion & & \\
Left ventricular end-diastolic pressure & $4.3 \pm 1.1$ & $5.0 \pm 1.3$ \\
$\quad$ Pressure (mm Hg) & $9.0 \pm 1.0^{*}$ & $8.6 \pm 3.3^{*}$ \\
Preocclusion & $2.87 \pm 0.20$ & $0.11 \pm .01 \ddagger$ \\
90 min of occlusion &
\end{tabular}

All values are presented as means \pm SE.

${ }^{*} P<.05$ from preocclusion values.

$\dagger P<.05$ from non-statin-treated group.

$\ddagger P=.0001$ from non-statin-treated group.

ings were reported by Weber and associates ${ }^{16}$ in a clinical study of patients with hypercholesterolemia. Lovastatin decreased CD11b-dependent adhesion of monocytes to human endothelium in patients independent of its cholesterol-lowering effects.

Statins generate mevalonate, a precursor that ultimately regulates the production of interleukin 8 (IL-8) and the proinflammatory cytokine IL-6. This ability of statins to reduce cell adhesiveness contributes to its reduction of ischemic events. Statins also contribute to reductions in ischemia-reperfusion injury by decreasing the inflammatory response associated with acute coronary syndromes. In the Pravastatin Inflammation C-Reactive Protein Evaluation study, patients treated with pravastatin after an MI or unstable angina had decreased levels of the inflammatory marker C-reactive protein. ${ }^{6}$ This effect occurred after only
12 weeks of therapy and was independent of changes in LDL levels.

The beneficial effects of statins in reducing ischemiareperfusion injury are not limited to the myocardium. Endres and coworkers ${ }^{8}$ studied the affects of statins in normocholesterolemic mice subjected to 2 hours of middle cerebral artery occlusion. Animals pretreated for 14 days with simvastatin had a reduction in cerebral infarct size by $46 \%$ and an increase in cerebral blood flow by $31 \%$. When animals were pretreated for only 3 days, the reduction in infarct size was decreased. Lower concentrations of simvastatin and the use of lovastatin were less effective. The decrease in infarct size was accompanied by the upregulation of endothelial NO synthase, which was independent of changes in serum cholesterol. The beneficial effects of simvastatin were absent in endothelial NO synthase-deficient 


\section{ENDOTHELIUM INDEPENDENT NITROGLYCERIN}

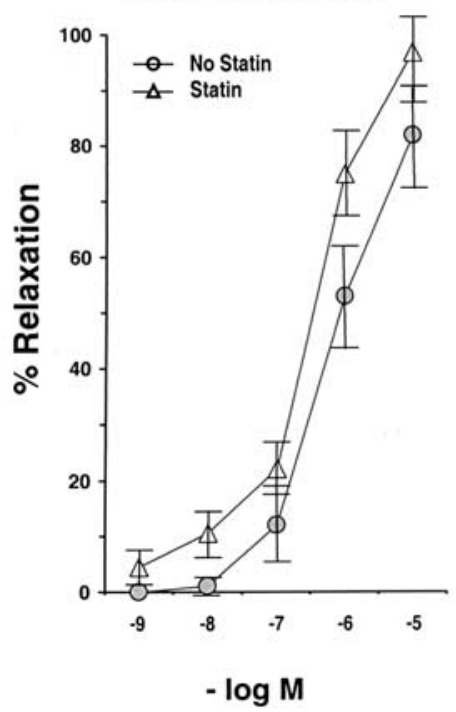

ENDOTHELIUM DEPENDENT

A23187

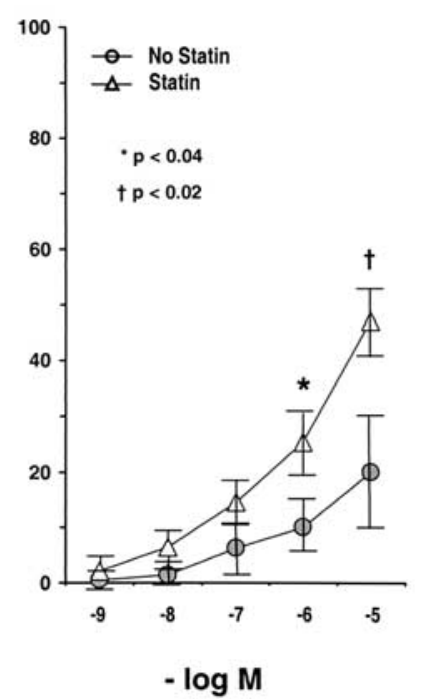

BRADYKININ

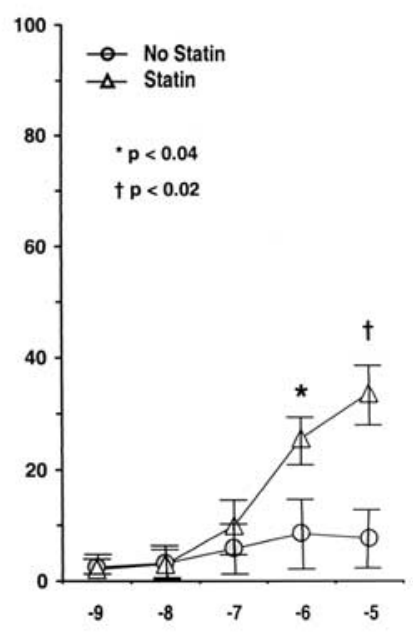

$-\log M$

Figure 2. Coronary artery relaxation. Endothelium-independent relaxation in response to nitroglycerin is preserved in both the statin-treated and non-statin-treated groups. Endothelium-dependent relaxation to A23187 and bradykinin is better preserved in the statin-treated group.

mice. The favorable effects of statins were also seen in normocholesterolemic rats with angiotensin II-induced hypertension. ${ }^{7}$ Cerivastatin decreased hypertension, cardiac hypertrophy, and fibrosis, suggesting that statins might also decrease $\mathrm{AT}_{1}$ receptor expression independent of their cholesterol-lowering properties.

The results of this experimental study suggest that pretreatment with statins might play an important role in reducing ischemia-reperfusion damage during coronary revascularization. However, in a recent clinical study, Florens and colleagues ${ }^{17}$ found no effect of atorvastatin in patients undergoing cardiac surgical procedures. In this study patients undergoing elective $\mathrm{CABG}$ and valve operations were given $40 \mathrm{mg}$ of atorvastatin in the evening before and $40 \mathrm{mg}$ the morning of the operation. All patients had normal serum cholesterol levels. There was no difference in clinical outcomes or in the expression of levels of inflammatory markers, such as CD11b, IL-6, IL-8, and P-selectin. There are, however, several explanations for the failure to observe any favorable effects of statin therapy in these patients. As noted by Endres and associates ${ }^{8}$ in his study on cerebral infarcts in mice, longer periods of statin therapy might be needed to see a reduction in ischemia-reperfusion injury. Furthermore, higher dosages might also be necessary. The MIRACL trial used $80 \mathrm{mg}$ of atorvastatin for a $70-\mathrm{kg}$ patient. ${ }^{4}$ The doses used in our own protocol for 35-kg animals were based on those used in the MIRACL trial. Hence the absence of any improvement in the study by Florens and colleagues ${ }^{17}$ might have been due to the short duration of therapy and the lower dose of the statin. In contrast, evidence for the clinical role of statin pretreatment before coronary revascularization was seen by Chan and coworkers ${ }^{18}$ in a retrospective study involving 5052 patients undergoing PTCA. In this series $26.5 \%$ of patients received statin therapy before their PTCA. Patients receiving statin therapy at the time of their PTCA had a significant reduction in mortality, which persisted for up to 6 months.

Our results suggest that statin therapy might offer additional protection to patients during coronary revascularization. However, several questions remain to be answered. How long will pretreatment with statin therapy be necessary, and can lower doses achieve the same anti-ischemic effects? Will all statin agents be equally effective in providing cardioprotection for surgical patients? These questions will need to be answered before conducting clinical trials and will be the focus of our future studies.

We thank Ms Ellie LaBombard and Ms Deborah Blackman for their assistance in preparing this manuscript.

\section{References}

1. Downs JR, Clearfield M, Weis S, Whitney E, Shapiro DR, Beere PA, et al. Primary prevention of acute coronary events with lovastatin in men and women with average cholesterol levels: results of AFCAPS/ TexCAPS Air Force/Texas Coronary Atherosclerosis Prevention Study. JAMA. 1998;279:1615-22.

2. Shepherd J, Cobbe SM, Ford I, Isles CG, Lorimer AR, MacFarlane PW, et al. Prevention of coronary heart disease with pravastatin in men with hypercholesterolemia. West of Scotland Coronary Prevention Study Group. N Engl J Med. 1995;333:401-7. 
3. The Long-Term Intervention with Pravastatin in Ischemic Disease (LIPID) Study Group. Prevention of cardiovascular events and death with pravastatin in patients with coronary heart disease and a broad range of initial cholesterol levels. $N$ Engl J Med. 1998;339:1349-57.

4. The Post Coronary Artery Bypass Graft Trial Investigators. The effect of aggressive lowering of low-density lipoprotein cholesterol levels and low-dose anticoagulation on obstructive changes in saphenousvein coronary-artery bypass grafts. $N$ Engl J Med. 1997;336:153-62.

5. Sacks FM, Pfeffer MA, Moye LA, Rouleau JL, Rutherford JD, Cole TG, et al. The effect of pravastatin on coronary events after myocardial infarction in patients with average cholesterol levels. Cholesterol and Recurrent Events Trial Investigators. N Engl J Med. 1995;335: 1001-9.

6. Albert MA, Danielson E, Rifa I. Effect of statin therapy on C-reactive protein levels: the Pravastatin Inflammation/CRP evaluation (PRINCE). A randomized trial and cohort study. JAMA. 2001;286:6470.

7. Dechend R, Fiebler A, Park J-K, Muller DN, Theuer J, Mervaala E, et al. Amelioration of angiotensin II-induced cardiac injury by a 3-hydroxy-3-methylglutaryl coenzyme A reductase inhibitor. Circulation. 2001;104:576-81.

8. Endres M, Laufs V, Huang Z, Nakamura T, Huang P, Moskowitz MA, et al. Stroke protection by 3-hydroxy-3-methylglutaryl (HMG)-CoA reductase inhibitors mediated by endothelial nitric oxide synthase. Proc Natl Acad Sci U S A. 1998;95:8880-5.

9. Lefer AM, Campbell B, Shin Y-K, Scalia R, Hayward R, Lefer DJ. Simvastatin preserves the ischemic-reperfused myocardium in normocholesterolemic rat hearts. Circulation. 1999;100:178-84.

10. Laufs V, La Fata V, Plutsky J, Liao JK. Upregulation of endothelial nitric oxide synthase by HMG CoA reductase inhibitors. Circulation. 1998;97:1129-35.

11. Schwartz GG, Oliver MF, Ezekowitz MD, Ganz P, Waters D, Kane JP, et al. Rationale and design of the Myocardial Ischemia Reduction with Aggressive Cholesterol Lowering (MIRACL) Study that evaluates atorvastatin in unstable angina pectoris and in non-Q wave acute myocardial infarction. Am J Cardiol. 1998;81:578-81.

12. Lazar HL, Yang XM, Rivers S, Treanor P, Shemin RJ. Role of percutaneous bypass in infarct size following revascularization for acute coronary insufficiency. Circulation. 1991;84(Suppl):III416-21.

13. Wagner $\mathrm{AH}$, Kohler $\mathrm{T}$, Ruckschloss V, Just I, Hecker M. Improvement in nitric oxide-dependent vasodilation by HMG-CoA reductase inhibitors through attenuation of endothelial superoxide anion formation. Arterioscler Thromb Vasc Biol. 2000;20:61-9.

14. Feron O, Dessy C, Desager JP, Belligard JL. HMG-CoA reductase inhibition promotes endothelial nitric oxide synthase activation through a decrease in caveolin abundance. Circulation. 2000;103: 113-8.

15. O'Driscoll G, Green D, Taylor RR. Simvastatin, an HMG-coenzyme A reductase inhibitor, improves endothelial function within 1 month. Circulation. 1997;95:1126-31.

16. Weber C, Erl W, Weber KJC, Weber PC. HMG-CoA reductase inhibitors decrease $\mathrm{CD} 11 \mathrm{~b}$ expression and $\mathrm{Cd} 11 \mathrm{~b}$-dependent adhesion of monocytes to endothelium and reduce increased adhesiveness of monocytes isolated from patients with hypercholesterolemia. $J \mathrm{Am}$ Coll Cardiol. 1997;30:1212-7.

17. Florens E, Salvi S, Peynet J, Elbim C, Mallat Z, Bel A, et al. Can statins reduce the inflammatory response to cardiopulmonary bypass? A Clinical Study. J Card Surg. 2001;16:232-9.

18. Chan AW, Bhatt DL, Chew DP, Quinn NJ, Moliterno DJ, Topol EJ, et al. Early and sustained survival benefit associated with statin therapy at the time of percutaneous coronary intervention. Circulation. 2002;105:691-6.

\section{Discussion}

Dr Henry M. Spotnitz (New York, NY). Do you want to speculate on what this might represent, and can you comment on whether there were any hemodynamic changes, heart rate changes, or any other ancillary differences you saw that might be involved in this?

Dr Lazar. During the 90-minute period of coronary occlusion, there were no significant hemodynamic changes. All the animals showed slightly decreased blood pressure, more so in the atorvastatin group than the others, but end-diastolic pressure was about the same. The mechanism can be divided into 3 properties that statins are known to have.

The first is improved endothelial function by enhanced stimulation of NO.

The second is decreased platelet thrombogenicity. Statins have been known, independent of their cholesterol-lowering effects, to have a favorable effect on P-selectin expression and CD18 expression, so that it is less likely for platelets to adhere to the arterial surface.

The third has to do with the inflammatory response. Statins have been shown in clinical studies after infarcts in patients undergoing bypass surgery to decrease C-reactive protein.

Dr Frank W. Sellke (Boston, Mass). Do you think 3 weeks is necessary? Could you give 2 days and get the same effect?

Dr Lazar. As you know, Phillipe Menasché did a clinical study in elective patients undergoing bypass surgery in which he gave his patients $40 \mathrm{mg}$ of atorvastatin the night before the operation and $40 \mathrm{mg}$ on the way to the operating room and found absolutely no change in any of the inflammatory markers. I think the reason for that is 2-fold.

First, he was looking at patients undergoing elective CABG, in which you would not expect to see much of a difference with any intervention. The second reason involves the dose and the timing. We have some data now to show that 1 week of $40 \mathrm{mg}$ of atorvastatin in this model has no therapeutic effect, but at 2 weeks with this same dose, there is a significant reduction in infarct size. Therefore, I would think that even at 10 days we might be able to see a beneficial effect.

The second question is, how much do you have to give? We gave $40 \mathrm{mg}$ to a $30-\mathrm{kg}$ animal, which would be the same as giving $80 \mathrm{mg}$ to a $70-\mathrm{kg}$ person, which is what was done in the MIRACL trial, a trial that showed improvement in patients receiving atorvastatin after an acute MI.

Therefore, I think this really needs to be worked out, but my feeling would be you do need to use higher doses, and you probably would have to treat them for anywhere between a week to 10 days.

Dr Sellke. All these patients should be receiving some sort of statin drug. If the primary care physicians and cardiologists were doing their job, this would not have as much clinical relevance, but it certainly does.

Dr Lazar. That is exactly right. All these patients who are at risk should be receiving a statin, regardless of their cholesterol level, and that includes the postoperative period. If they are taking a statin and we know that we are going to be doing an operation, they should stay on it and perhaps their dosage should be increased. 